\title{
Editorial for EAIT Issue 6, 2017
}

\author{
Arthur Tatnall ${ }^{1}$
}

Published online: 7 November 2017

(C) Springer Science+Business Media, LLC 2017

This issue of the journal contains fifteen regular articles and a Special Edition section titled: Students, Computers and Learning: Where Is the Connection? This has been Guest Edited by Chenglie Hu from Carroll University, USA.

The first of the regular articles in this issue is by FatimaEzzahra Benmarrakchi, Jamal El Kafi and Ali Elhore (Chouaib Doukkali University, El Jadida, Morocco) and Sara Haie (International University of Casablanca, Morocco). Titled: Exploring the use of the ICT in supporting dyslexic students' preferred learning styles: A preliminary evaluation it begins by pointing out that dyslexia is one of the most common learning disabilities, affecting $7-10 \%$ of the population across most languages and cultures, and is manifested by difficulty in learning to read despite conventional instruction, adequate intelligence, and sociocultural opportunity (https://doi.org/10.1007/s10639-016-95514). Their study explores the potential benefits offered by the use of Information and Communication Technology to support dyslexic students by considering their preferred learning styles. Their study focused on dyslexia in Arabic language, especially in Arabic countries such as Morocco.

Next is an article titled: Wikipedia as a platform for impactful learning: A new course model in higher education and has been contributed by Shani Evenstein Sigalov and Rafi Nachmias (Tel Aviv University, Israel) (https://doi.org/10.1007/s10639-0169564-z). This article investigates the design and implementation of a course dedicated to Wikipedia. Learning focused on improved consumption of information and collaborative construction of knowledge using the Wikipedia platform. As well as medical articles in Hebrew Wikipedia, students reported a unique learning experience that sharpened their collaborative skills as well as academic skills.

Following is: Technology enhanced learning in programming courses -an international perspective by Mirjana Ivanović (University of Novi Sad, Serbia), Stelios Xinogalos (University of Macedonia, Greece), Tomáš Pitner (Masaryk University, Czech Republic) and Miloš Savić (University of Novi Sad, Serbia) (https://doi. org/10.1007/s10639-016-9565-y). They note that technology enhanced learning is

Arthur Tatnall

Arthur.Tatnall@vu.edu.au

1 Victoria University, PO Box 14428, Melbourne 8001, Australia 
increasingly influencing university education by overcoming disadvantages of direct instruction teaching approaches, and encouraging creativity, problem solving and critical thinking in student-centred, interactive learning environments. In this article they discuss and compare experiences from object-oriented programming courses taught in three institutions from three different European countries.

An online authoring tool for creating activity-based learning objects by Jeong Yong Ahn, Gil Seong Mun and Kyung Soo Han (Chonbuk National University, South Korea) and Sook Hee Choi (Woosuk University, South Korea) follows (https://doi.org/10.1007 /s10639-016-9567-9). As higher education increasingly relies on e-learning, the need for tools that will allow teachers themselves to develop effective e-learning objects as simply and quickly as possible has also been increasingly recognized. This article discusses the design and development of a novel tool, Enook (Evolutionary note book), for creating activity-based learning objects (LOs).

The next article: LTSA conformance testing to architectural design of LMS using ontology was contributed by Souvik Sengupta (Bengal Institute of Technology, Kolkata, India) and Ranjan Dasgupta (National Institute of Technical Teachers' Training and Research, Kolkata, India) (https://doi.org/10.1007/s10639-016-9569-7). Their article proposes a new methodology for checking conformance of the software architectural design of Learning Management System (LMS) to Learning Technology System Architecture (LTSA). In their approach, the architectural designing of LMS follows the formal modelling style of Acme and an ontology is built to represent the LTSA rules and the software architecture in Acme style.

The effect of badges on the engagement of students with special educational needs: A case study, by Ourania Sitra, Vangelis Katsigiannakis and Charalampos Karagiannidis (University of Thessaly, Greece) and Sofia Mavropoulou (University of New England, Australia) addresses the perceived benefits from gamification in the context of special education and presents findings of a study evaluating the effects of a specific gamification element (badges) on the engagement of five students with special learning needs, through online courses developed on the Moodle Learning Management System (LMS) (https://doi.org/10.1007/s10639-016-9550-5). The results indicate that this particular gamification element yielded positive effects on students' engagement and on their overall attitude towards the educational process in general.

What follows is an article by: J. Petrović, P. Pale and B. Jeren (University of Zagreb, Croatia). Online formative assessments in a digital signal processing course: Effects of feedback type and content difficulty on students learning achievements describes a study aimed to investigate the effects of using online formative assessments on students' learning achievements (https://doi.org/10.1007/s10639-016-9571-0). Their study findings suggest online formative assessments are a very efficient educational intervention for this domain. These are significant for demonstrating the potential of online formative assessments in achieving the desired learning outcomes in higher education, as well as for gaining insights into students' habits of using them.

The next article discusses a topic of interest to all educators: Smartphones usage in the classrooms: Learning aid or interference? The authors: Muhammad Anshari, Mohammad Nabil Almunawar and Masitah Shahrill (Universiti Brunei Darussalam, Brunei), Danang Kuncoro Wicaksono (Islam Sultan Sharif Ali, Brunei) and Miftachul Huda (Universiti Teknologi Malaysia, Malaysia) note that many educational institutions, especially higher education institutions, are embracing smartphones as learning 
aids (https://doi.org/10.1007/s10639-017-9572-7). The question is whether embracing smartphones in classroom teaching enhances learning or perhaps interferes.

Blurring time and place in higher education with bring your own device applications: a literature review is by Marcus Sundgren (Mid Sweden University, Sweden) (https://doi.org/10.1007/s10639-017-9576-3). The use of mobile devices is increasing rapidly in society, and student device ownership is becoming ubiquitous in many parts of the world which might be an under-utilised resource that could benefit the educational practices of institutions of higher education. This review examines 91 journal articles from 28 countries published in the years of 2009-2015 with regards to the applications of Bring Your Own Device (BYOD) in higher education to take inventory of how it is applied where blurring of boundaries of time and place can be observed, and to observe problems or obstacles regarding these applications.

Aditya Khamparia and Babita Pandey (Lovely Professional University, India) then offer: Comprehensive analysis of semantic web reasoners and tools: a survey (https://doi.org/10.1007/s10639-017-9574-5). They begin by claiming that ontologies are emerging as the best representation techniques for knowledge-based context domains and that the continuing need for interoperation, collaboration and effective information retrieval has led to the creation of the semantic web with tools and that manage personalised information. Their article compares the latest and the traditional reasoners like Pellet, RACER, HermiT, FaCT++ with respect to features supported by them. Their research involved survey and comparison of numerous reasoning models, ontology tools and web services.

Designing and creating an educational app rubric for preschool teachers by Stamatios Papadakis, Michail Kalogiannakis and Nicholas Zaranis (University of Crete, Greece) points out that international studies indicate that the use of smart mobile devices and their accompanying educational apps can revolutionize young children's learning experiences (https://doi.org/10.1007/s10639-017-9579-0). It is important for preschool teachers to be able to assess each app for its effectiveness in educational practice and to evaluate educational mobile apps this article presents a rubric (abbreviated as REVEAC) in four areas: contents, design, functionality, and technical quality, each having multiple aspects.

The article that follows: Digital literacy and online video: Undergraduate students' use of online video for coursework investigates how to enable undergraduate students' use of online video for coursework using a customised video retrieval system (VRS), in order to understand digital literacy with online video in practice (https://doi. org/10.1007/s10639-017-9575-4). It describes research by Peter Tiernan and Margaret Farren (Dublin City University, Ireland). Their study examines key areas influencing the use of online video for assignments such as the learning value of video, strategies for its integration and the key features of online video systems. It examines how students source, integrate, and reference online video for assignment work.

Suad Abdul Aziz Al-Furai (Kuwait University, State of Kuwait) next explores the perceptions of pre-service teachers on the design of a learning environment using the Seven Principles of Good Practice and its effect on participants' abilities to create their Cloud Learning Environment (CLE). Perceptions of pre-service teachers on the design of a learning environment based on the seven principles of good practice produced findings that indicated the 7P-ILD positively influenced participants' ability to confidently build their CLE and that participants were most satisfied with 7P-ILD related to 
the principle student-faculty contact, and least satisfied with the principle time-on-task (https://doi.org/10.1007/s10639-017-9580-7).

An empirical verification of a-priori learning models on mailing archives in the context of online learning activities of participants in freellibre open source software (FLOSS) communities is from Patrick Mukala (University of Pisa, Italy), Antonio Cerone (Nazarbayev University, Kazakhstan) and Franco Turini (University of Pisa, Italy) (https://doi.org/10.1007/s10639-017-9573-6). They note that FreelLibre Open Source Software (FLOSS) environments are increasingly dubbed as learning environments where practical software engineering skills can be acquired and that numerous studies have extensively investigated how knowledge is acquired in these environments through a collaborative learning model that define a learning process. Their article analyses a-priori learning models in comparison with the real behaviour captured in FLOSS repositories by means of conformance verification in process mining.

The last article in this issue: Copyright ownership of e-learning and teaching materials: Policy approaches taken by UK universities, comes from Elizabeth Gadd (Loughborough University, UK) and Ralph Weedon (University of Strathclyde, UK) (https://doi.org/10.1007/s10639-017-9583-4). Their article investigates whether and how UK university copyright policies address key copyright ownership issues relating to printed and electronic teaching materials. Their article recommends that UK universities work with academic staff to address key copyright policy issues in a way that balances the rights of both parties.

\section{Arthur Tatnall}

EAIT Editor-in-Chief 